\title{
A remark on a paper by Evans and Harris on the point spectra of Dirac operators
}

\author{
Karl Michael Schmidt \\ School of Mathematics, Cardiff University, \\ 23 Senghennydd Road, Cardiff CF24 4YH, UK \\ (MS received 19 June 2000; accepted 6 December 2000) \\ This paper presents a sufficient condition for a one-dimensional Dirac operator with a \\ potential tending to infinity at infinity to have no eigenvalues. It also provides a \\ quick proof (and suggests variations) of a related criterion given by Evans and Harris.
}

In [3], Evans and Harris obtained bounds for regions in the real line that cannot contain eigenvalues of one-dimensional Dirac operators. In the special case of the Dirac operator

$$
-\mathrm{i} \sigma_{2} \frac{\mathrm{d}}{\mathrm{d} x}+\sigma_{3}+p \sigma_{1}+w+r
$$

with a potential $w$ tending to infinity at infinity and asymptotically dominating the angular momentum term $p$ and the perturbation $r$, they have given the following sufficient criterion for the non-existence of eigenvalues altogether (see [3, theorem 3]).

Theorem 1.1. Let $w, p \in A C_{\mathrm{loc}}[a, \infty), r \in L_{\mathrm{loc}}^{1}[a, \infty), a>0$. Suppose that

$$
\begin{aligned}
\lim _{x \rightarrow \infty}|w(x)| & =\infty, \\
\limsup _{x \rightarrow \infty}\left|\frac{p(x)}{w(x)}\right| & =0, \\
\limsup _{x \rightarrow \infty} \frac{1}{\log x} \int_{a}^{x} \frac{\left(w^{\prime}\right)_{-}}{|w|^{2}} & =: L_{1}, \\
\limsup _{x \rightarrow \infty} \frac{1}{\log x} \int_{a}^{x}\left|\frac{r}{w}\right| & =: K_{1}, \\
\lim _{x \rightarrow \infty} \frac{1}{\log x} \int_{a}^{x}\left(\left|\frac{p r}{w}\right|+\left|\frac{p w^{\prime}}{w^{2}}\right|+\left|\frac{p^{\prime}}{w}\right|\right) & =0 .
\end{aligned}
$$

Then there are no non-trivial $L^{2}(a, \infty)$ solutions of

$$
\left(-\mathrm{i} \sigma_{2} \frac{\mathrm{d}}{\mathrm{d} x}+\sigma_{3}+p \sigma_{1}+w+r\right) u=\lambda u
$$

for any $\lambda \in \mathbb{R}$ if $L_{1}+K_{1}<\frac{1}{2}$.

The observation that the one-dimensional Dirac operator (with $p \equiv r \equiv 0$ ) has a purely continuous spectrum, even covering the whole real line, if $w$ tends to infinity 
at infinity and satisfies certain conditions, goes back to Plesset [7] in the case of polynomial $w$. As stated correctly, though with an incorrect proof, by Rose and Newton [9], it is sufficient to assume that $w$ is eventually non-decreasing.

In their study of the spectrum in a variety of cases for the asymptotic behaviour of the coefficients, Roos and Sangren [8] note 'continuous spectrum $-\infty<\lambda<\infty$ ' if (3.16) holds, corresponding to their cases (3) and (4); a statement of appealing generality but unfortunately false, as (3.16) alone is compatible both with the existence of eigenvalues (cf. [12]) and of gaps in the essential spectrum (cf. [13]). Indeed, in the proof they observe that 'it is advantageous to assume further [...] that $S(t)$ is $L(0, \infty)$ ', where in the case at hand

$$
S(t) \sim \frac{w^{\prime 2}(t)}{w^{3}(t)}-\frac{w^{\prime \prime}(t)}{2 w^{2}(t)}, \quad t \rightarrow \infty
$$

(note that there is a misprint in formula (6) of [8], which should read $G(t, \lambda)=$ $\left.\left(\alpha^{\prime}\right)^{-1} F^{\prime}(t, \lambda)\right)$. Thus the actual hypotheses of [8] coincide with Titchmarsh's criterion for purely absolutely continuous spectrum covering the whole real line [15], subsequently weakened to

$$
w \in A C_{\mathrm{loc}}, \quad \int^{\infty} \frac{\left|w^{\prime}\right|}{w^{2}}<\infty
$$

by Erdélyi [2] (cf. [12]).

For the more general question of ruling out the existence of eigenvalues only, Erdélyi's criterion can in turn be refined, as shown in theorem 1.1 above.

In this paper we present a transparent approach to this case, which easily yields variants of the Evans-Harris criterion. In particular, we weaken the regularity requirement on the potential to locally bounded variation, which in the light of similar results (see $[12,14,17]$ and $[16, \S 8]$ ) appears to be the natural setting for this kind of question. Our fundamental result is the following.

Theorem 1.2. Let $M, M_{1}, Q, Q_{1} \in L_{\mathrm{loc}}^{1}(\cdot, \infty)$ be real-valued functions such that

$$
M \geqslant 0, \quad \lim _{x \rightarrow \infty} Q(x)=\infty, \quad \limsup _{x \rightarrow \infty} \frac{M(x)}{Q(x)}<1 \quad \text { and } \quad \frac{M}{Q-M} \in B V_{\mathrm{loc}}(\cdot, \infty) .
$$

Then the Dirac system

$$
\left(-\mathrm{i} \sigma_{2} \frac{\mathrm{d}}{\mathrm{d} x}+\left(M+M_{1}\right) \sigma_{3}+Q+Q_{1}\right) u=0
$$

has no non-trivial solution $u \in L^{2}(\cdot, \infty)$ if

$$
\int^{\infty} \exp \left(-2 P\left(\frac{M}{Q-M}\right)(t)-2 \int^{t} \frac{\left|M_{1} Q-M Q_{1}\right|}{Q-M}\right) \mathrm{d} t=\infty .
$$

Here we use the notation $X(\cdot, \infty):=\{u \mid$ there is $a \in \mathbb{R}$ such that $u \in X(a, \infty)\}$, with $X$ any symbol for a space of functions on a real interval.

Furthermore, if $f$ is a function of locally bounded variation $\left(B V_{\text {loc }}\right)$, we denote by $P f$ its positive variation, defined up to an additive constant by

$$
P f(b)-P f(a)=\sup \sum_{j=1}^{n}\left(f\left(x_{j}\right)-f\left(x_{j-1}\right)\right)_{+},
$$


where the supremum is taken over all partitions $a=x_{0}<x_{1}<\cdots<x_{n}=b$, $n \in \mathbb{N}$.

The Dirac operator (1.1) has a matrix term $p \sigma_{1}$, and thus does not immediately satisfy the hypotheses of theorem 1.2. Nevertheless, if we assume $p$ to be locally absolutely continuous, it is unitarily equivalent to the Dirac operator

$$
-\mathrm{i} \sigma_{2} \frac{\mathrm{d}}{\mathrm{d} x}+\sqrt{1+p^{2}} \sigma_{3}+w+r-\frac{p^{\prime}}{2\left(1+p^{2}\right)}
$$

by virtue of the transformation given in [11, p. 467] (cf. however, remark 1.7 below).

Thus, taking in theorem 1.2 ,

$$
M:=1, \quad M_{1}:=\sqrt{1+p^{2}}-1 \leqslant p^{2}, \quad Q:=w-\lambda \quad \text { and } \quad Q_{1}:=r-\frac{p^{\prime}}{2\left(1+p^{2}\right)},
$$

we obtain the following result.

Corollary 1.3. Let $w, r \in L_{\mathrm{loc}}^{1}(\cdot, \infty), p \in A C_{\mathrm{loc}}(\cdot, \infty)$ be real-valued functions such that $\lim _{x \rightarrow \infty} w(x)=\infty$ and $1 / w \in B V_{\mathrm{loc}}(\cdot, \infty)$. Then (1.2) has no non-trivial solution $u \in L^{2}(\cdot, \infty)$ if

$$
\limsup _{x \rightarrow \infty} \frac{1}{\log x}\left(P\left(\frac{1}{w}\right)(x)+\int^{x}\left(\frac{|r|}{w}+\frac{\left|p^{\prime}\right|}{2 w}+p^{2}\right)\right)<\frac{1}{2} .
$$

The constant $\frac{1}{2}$ is optimal in the sense that for each $\alpha>\frac{1}{2}$, there is a potential $w$ such that

$$
\limsup _{x \rightarrow \infty} \frac{P(1 / w)(x)}{\log x}<\alpha,
$$

and the Dirac operator (1.1), with $p \equiv r \equiv 0$, has an eigenvalue. Indeed, a piecewise constant $w$ with these properties can easily be obtained from the example given in $[12, \S 3]$ by increasing the lengths of the intervals on which the potential is constant.

If, in addition to the hypotheses of corollary 1.3 , we assume $w \in A C_{\text {loc }}(\cdot, \infty)$, then

$$
P\left(\frac{1}{w}\right)(x)=\int^{x} \frac{\left(w^{\prime}\right)_{-}}{w^{2}}
$$

and $\lim _{x \rightarrow \infty}|w(x)|=\infty$ means either $w(x) \rightarrow \infty$ or $w(x) \rightarrow-\infty$, which is essentially the same, due to the inherent symmetry of the Dirac system. Thus the above conditions on $w$ and $r$ correspond precisely to (3.16), (3.18) and (3.19).

However, corollary 1.3, though in itself presenting a criterion of appealing simplicity for the absence of eigenvalues, does not entail theorem 1.1. Indeed, corollary 1.3 includes an integral condition on $p^{2}$, whereas (3.20) only poses conditions on the size of $p, p^{\prime}$ relative to that of $w$.

But then, taking $Q$ and $Q_{1}$ as above, but $M:=\sqrt{1+p^{2}}, M_{1}:=0$ in theorem 1.2, we obtain the following mild generalization of theorem 1.1.

Corollary 1.4. Let $r \in L_{\mathrm{loc}}^{1}(\cdot, \infty), w, p \in A C_{\mathrm{loc}}(\cdot, \infty)$ be real-valued functions such that

$$
\lim _{x \rightarrow \infty} w(x)=\infty \quad \text { and } \quad \lim _{x \rightarrow \infty} \frac{|p|}{w}(x)=0 .
$$


Then (1.2) has no non-trivial solution $u \in L^{2}(\cdot, \infty)$ if

$$
\limsup _{x \rightarrow \infty} \frac{1}{\log x} \int^{x}\left(\left(\frac{\left(w^{\prime}\right)_{-}}{w^{2}}+\frac{|r|}{w}\right)(1+|p|)+\frac{3\left|p^{\prime}\right|}{2 w}\right)<\frac{1}{2} .
$$

REMARK 1.5. In the case of the angular momentum term arising from a separation in polar coordinates of a higher-dimensional rotationally symmetric Dirac operator, $p(x)=k / x$,

$$
\frac{\left|p^{\prime}\right|}{2 w}+p^{2}=\left(\frac{|k|}{2 w}+k^{2}\right) \frac{1}{x^{2}}
$$

will be integrable at $\infty$, so that the condition of corollary 1.3 reduces to

$$
\limsup _{x \rightarrow \infty} \frac{1}{\log x}\left(P\left(\frac{1}{w}\right)(x)+\int^{x} \frac{|r|}{w}\right)<\frac{1}{2} .
$$

Likewise, corollary 1.4 has this condition with the positive variation expressed as the integral of the positive part of the derivative.

We also remark that in view of $\lim _{x \rightarrow \infty}|w(x)|=\infty$, equation (3.18) already implies

$$
\limsup _{x \rightarrow \infty} \frac{1}{\log x} \int_{a}^{x} \frac{\left|w^{\prime}\right|}{w^{2}}=2 L_{1}
$$

and hence

$$
\lim _{x \rightarrow \infty} \frac{1}{\log x} \int_{a}^{x} \frac{\left|w^{\prime}(t)\right|}{t w^{2}(t)} \mathrm{d} t=0 .
$$

Therefore, hypothesis (3.20) is satisfied if $p(x)=k / x$, without the additional assumption (3.23) introduced in [3, remark 4].

The idea of the proof of theorem 1.2 is to make the method of $[12,14]$ quantitative. One of the key observations of these studies (see [12, lemma 2]) shows that a locally bounded function satisfying a rough Gronwall-type estimate with a righthand side resembling a Stieltjes integral with a bounded integrator, is bounded; this was used to give a sufficient condition for all solutions of Dirac systems with a divergent potential to be bounded. In the present situation, we similarly exclude the existence of $L^{2}$ solutions by estimating the growth of solutions and applying a standard Wronskian argument.

The Gronwall lemma has been generalized to Stieltjes integrals in various ways before (cf. $[1,4,6,10])$; however, these extensions do not quite meet our present requirements. The following refinement of [12, lemma 2$]$ is readily proved along the lines of $[5$, theorem 1.4$]$.

Lemma 1.6. Let $c \in \mathbb{R}, C>0$ and $f:[c, \infty) \rightarrow[0, \infty)$ be a continuous function satisfying

$$
f(t) \leqslant C+\int_{c}^{t} f(s) \mathrm{d} \alpha(s), \quad t \geqslant c,
$$

with non-decreasing $\alpha:[c, \infty) \rightarrow \mathbb{R}, \alpha(c)=0$.

Then $f(t) \leqslant C \exp (\alpha(t)), t \geqslant c$. 
Proof of theorem 1.2. As the Dirac system (1.3) has real coefficients, it is clearly sufficient to consider $\mathbb{R}^{2}$-valued solutions.

Let $u$ be a solution, and $v$ a linearly independent solution, of (1.3).

As $Q-M=Q(1-M / Q) \rightarrow \infty$ at $\infty$, we can define

$$
R:=\frac{\left(v_{1}^{2}+v_{2}^{2}\right) Q+\left(v_{1}^{2}-v_{2}^{2}\right) M}{Q-M} \in B V_{\mathrm{loc}}(\cdot, \infty) .
$$

Then $R \geqslant|v|^{2}$, and for sufficiently large $t_{1} \leqslant t_{2}$ we have, by the rule of integration by parts for Stieltjes integrals,

$$
\begin{aligned}
R\left(t_{2}\right)-R\left(t_{1}\right) & =\int_{t_{1}}^{t_{2}}\left(|v|^{2}\right)^{\prime}+\int_{t_{1}}^{t_{2}} \frac{2 M}{Q-M}\left(v_{1}^{2}\right)^{\prime}+\int_{t_{1}}^{t_{2}} 2 v_{1}^{2} \mathrm{~d}\left(\frac{M}{Q-M}\right) \\
& =\int_{t_{1}}^{t_{2}} 4 v_{1} v_{2} \frac{Q M_{1}-M Q_{1}}{Q-M}+\int_{t_{1}}^{t_{2}} 2 v_{1}^{2} \mathrm{~d}\left(\frac{M}{Q-M}\right) \\
& \leqslant 2 \int_{t_{1}}^{t_{2}}|v|^{2} \frac{\left|Q M_{1}-M Q_{1}\right|}{Q-M}+2 \int_{t_{1}}^{t_{2}} v_{1}^{2} \mathrm{~d} P\left(\frac{M}{Q-M}\right) .
\end{aligned}
$$

Hence

$$
|v|^{2}\left(t_{2}\right) \leqslant R\left(t_{2}\right) \leqslant R\left(t_{1}\right)+\int_{t_{1}}^{t_{2}}|v|^{2} \mathrm{~d} \alpha
$$

where

$$
\alpha(t):=2 \int_{t_{1}}^{t} \frac{\left|Q M_{1}-M Q_{1}\right|}{Q-M}+2 P\left(\frac{M}{Q-M}\right)(t)-2 P\left(\frac{M}{Q-M}\right)\left(t_{1}\right), \quad t \geqslant t_{1} .
$$

By the above Lemma, we conclude $|v|^{2}(t) \leqslant R\left(t_{1}\right) \exp (\alpha(t)), t \geqslant t_{1}$.

If $W$ is the Wronskian of $u, v$, the estimate $|u|^{2}|v|^{2}=W^{2}+\left(u_{1} v_{1}+u_{2} v_{2}\right)^{2} \geqslant W^{2}$ implies

$$
\int_{t_{1}}^{\infty}|u|^{2} \geqslant W^{2} \int_{t_{1}}^{\infty} \frac{1}{|v|^{2}} \geqslant \frac{W^{2}}{R\left(t_{1}\right)} \int_{t_{1}}^{\infty} \exp (-\alpha)=\infty
$$

Proof of corollary 1.3. With the choices for $M, M_{1}, Q, Q_{1}$ indicated before the statement of corollary 1.3, we find

$$
\begin{aligned}
P\left(\frac{M}{Q-M}\right)(t)+\int^{t} \frac{\left|M_{1} Q-M Q_{1}\right|}{Q-M} & \\
& \leqslant P\left(\frac{1}{w-\lambda-1}\right)+\int^{t} \frac{p^{2}(w-\lambda)+|r|+\frac{1}{2}\left|p^{\prime}\right|}{w-\lambda-1} .
\end{aligned}
$$

For any $\lambda \in \mathbb{R}$ and sufficiently large $x, y \in \mathbb{R}$, we have

$$
\left(\frac{1}{w(x)-\lambda-1}-\frac{1}{w(y)-\lambda-1}\right)_{+}=\frac{w(x)}{w(x)-\lambda-1} \frac{w(y)}{w(y)-\lambda-1}\left(\frac{1}{w(x)}-\frac{1}{w(y)}\right)_{+},
$$


and thus the positive variation on an interval $[s, t]$ satisfies

$$
\begin{aligned}
P\left(\frac{1}{w-\lambda-1}\right)(t)- & P\left(\frac{1}{w-\lambda-1}\right)(s) \\
& \leqslant\left(1+\sup _{[s, t]} \frac{\lambda-1}{w-\lambda-1}\right)^{2}\left(P\left(\frac{1}{w}\right)(t)-P\left(\frac{1}{w}\right)(s)\right) .
\end{aligned}
$$

Thus we have

$$
\begin{aligned}
P\left(\frac{1}{w-\lambda-1}\right)(t) & -P\left(\frac{1}{w-\lambda-1}\right)(s)+\int_{s}^{t} \frac{p^{2}(w-\lambda)+|r|+\frac{1}{2}\left|p^{\prime}\right|}{w-\lambda-1} \\
& \leqslant\left(P\left(\frac{1}{w}\right)(t)-P\left(\frac{1}{w}\right)(s)+\int_{s}^{t}\left(p^{2}+\frac{|r|+\frac{1}{2}\left|p^{\prime}\right|}{w}\right)\right)(1+o(1))
\end{aligned}
$$

as $s \rightarrow \infty$ and, taking $s$ large enough,

$$
\limsup _{t \rightarrow \infty} \frac{1}{\log t}\left(P\left(\frac{1}{w-\lambda-1}\right)(t)+\int^{t} \frac{p^{2}(w-\lambda)+|r|+\frac{1}{2}\left|p^{\prime}\right|}{w-\lambda-1}\right)<\frac{1}{2} .
$$

Consequently,

$$
\int^{\infty} \exp \left(-2 P\left(\frac{M}{Q-M}\right)(t)-2 \int^{t} \frac{\left|M_{1} Q-M Q_{1}\right|}{Q-M}\right) \mathrm{d} t>\int^{\infty} \frac{\mathrm{d} t}{t}=\infty,
$$

which, by theorem 1.2 , implies the non-existence of $L^{2}(\cdot, \infty)$ solutions.

Proof of corollary 1.4. With the choices for $M, M_{1}, Q, Q_{1}$ indicated before the statement of corollary 1.4, we find

$$
\begin{aligned}
P\left(\frac{M}{Q-M}\right)(t)+\int^{t} \frac{\left|M_{1} Q-M Q_{1}\right|}{Q-M} & \\
=\int^{t}\left(\left(\frac{p p^{\prime}}{\sqrt{1+p^{2}}\left(w-\lambda-\sqrt{1+p^{2}}\right)}-\right.\right. & \left.\frac{\sqrt{1+p^{2}} w^{\prime}-p p^{\prime}}{\left(w-\lambda-\sqrt{1+p^{2}}\right)^{2}}\right)_{+} \\
& \left.+\frac{\sqrt{1+p^{2}}\left|r-p^{\prime} / 2\left(1+p^{2}\right)\right|}{\left(w-\lambda-\sqrt{1+p^{2}}\right)}\right) .
\end{aligned}
$$

The integrand has the upper bound

$$
\left(\frac{3\left|p^{\prime}\right|}{2 w}+(1+|p|)\left(\frac{\left(w^{\prime}\right)_{-}}{w^{2}}+\frac{|r|}{w}\right)\right)(1+o(1)),
$$

and the assertion follows as in the proof of corollary 1.3.

REMARK 1.7. In order to remove the matrix term $p \sigma_{1}$ from the original Dirac operator (1.1) by a unitary transformation, we had to assume that $p$ be locally absolutely continuous. This restriction can be avoided by treating the full system (1.2) directly, as in the proof of [14, proposition 2]. The resulting sufficient condition for the absence of $L^{2}(\cdot, \infty)$ solutions involves the variations of various combinations of the coefficients only; however, when $w, p$ are locally absolutely continuous, this does not improve upon corollary 1.4. 


\section{Acknowledgments}

I thank the referee for pointing out reference [8].

\section{References}

1 L. H. Erbe and Q. K. Kong. Stieltjes integral inequalities of Gronwall type and applications. Annli Mat. Pura Appl. 157 (1990), 77-97.

2 A. Erdélyi. Note on a paper by Titchmarsh. Q. J. Math. Oxf. 14 (1963), 147-152.

3 W. D. Evans and B. J. Harris. Bounds for the point spectra of separated Dirac systems. Proc. R. Soc. Edinb. A 88 (1981), 1-15.

4 J. V. Herod. A Gronwall inequality for linear Stieltjes integrals. Proc. Am. Math. Soc. 23 (1969), 34-36.

5 D. B. Hinton. A Stieltjes-Volterra integral equation theory. Can. J. Math. 18 (1966), 314331 .

6 A. B. Mingarelli. On a Stieltjes version of Gronwall's inequality. Proc. Am. Math. Soc. 82 (1981), 249-252.

7 M. S. Plesset. The Dirac electron in simple fields. Phys. Rev. 41 (1932), 278-290.

8 B. W. Roos and W. C. Sangren. Spectra for a pair of singular first order differential equations. Proc. Am. Math. Soc. 12 (1961), 468-476.

9 M. E. Rose and R. R. Newton. Properties of Dirac wave functions in a central field. Phys. Rev. 82 (1951), 470-477.

10 W. W. Schmaedeke and G. R. Sell. The Gronwall inequality for modified Stieltjes integrals. Proc. Am. Math. Soc. 19 (1968), 1217-1222.

11 K. M. Schmidt. Dense point spectrum and absolutely continuous spectrum in spherically symmetric Dirac operators. Forzm Math. 7 (1995), 459-475.

12 K. M. Schmidt. Absolutely continuous spectrum of Dirac systems with potentials infinite at infinity. Math. Proc. Camb. Phil. Soc. 122 (1997), 377-384.

13 K. M. Schmidt. A remark on the essential spectra of Dirac systems. Bull. Lond. Math. Soc. 32 (2000), 63-70.

14 K. M. Schmidt and O. Yamada. Spherically symmetric Dirac operators with variable mass and potentials infinite at infinity. Proc. RIMS, Kyoto Univ. 34 (1998), 211-227.

15 E. C. Titchmarsh. On the nature of the spectrum in problems of relativistic quantum mechanics. Q. J. Math. Oxf. 12 (1961), 227-240.

16 J. Weidmann. Oszillationsmethoden für Systeme gewöhnlicher Differentialgleichungen. Math. Z. 119 (1971), 349-373.

17 J. Weidmann. Absolutstetiges Spektrum bei Sturm-Liouville-Operatoren und Diracsystemen. Math. Z. 180 (1982), 423-427.

(Issued 19 October 2001) 